\title{
Solid waste indicators in local sustainability assessment: a literature review
}

\author{
Natália Molina Cetrulo \\ Tiago Balieiro Cetrulo \\ Sylmara Lopes Francelino Gonçalves Dias \\ Tomás Barros Ramos
}

${ }^{1}$ School of Arts, Sciences and Humaniyies, University of São Paulo. São Paulo, SP - Brazil.

(D) II São Carlos School of Engineering, University of São Paulo. São Carlos, SP - Brazil.

III School of Arts, Sciences and Humaniyies, University of São Paulo. São Paulo, SP - Brazil.

IV CENSE - Center for Environmental and Sustainability Research. NOVA School of Science and Technology. NOVA University Lisbon. Caparica - Portugal.

\begin{abstract}
The Solid Waste (SW) topic within the local sustainability assessment (LSA) system context remains little explored. This article is a literature review on this topic, with emphasis on the analysis of SW indicators and of systems they concern to. It assessed whether these indicators can represent SW complexity and sustainability aspects. Results in the present review have evidenced that $77.8 \%$ of system indicators use at most two SW indicators. Most of these indicators aim at indicating destination stages and the final SW management, environmental sustainability aspects and intermediate magnitude levels in SW management hierarchy. There was evidence of the positive impact of Public participation on the assessed matters. Final considerations present recommendations about SW indicators inclusion in LSA systems.
\end{abstract}

Keywords: Sustainability indicators; Local Sustainability assessment; Public participation; Waste management hierarchy.

São Paulo. Vol. 23, 2020

Original Article

DOI: http://dx.doi.org/10.1590/1809-4422asoc20190028r3vu2020L5AO 


\section{Introduction}

Population growth, increased urbanization rate and changes in society's consumption patterns lead to increased solid waste generation (ESMAEILIAN et al., 2018). Such large population, added with variability and diversification in waste composition, has complex and localized effect on sustainability, since it covers social, environmental, governance and management aspects, as well as shows great variability from location to location (BURNLEY, 2007; GUERRERO; MAAS; HOGLAND, 2013). Accordingly, it is essential to add solid waste management aspects to LSA tools.

The literature presents a series of local sustainability indicator systems that encompass SWs, such as systems developed by Lee and Huang (2007), Nader, Salloum and Karam (2008) and Moreno-Pires, Fidélis and Ramos (2014). In these cases, SW indicators are part of the assessment system, as well as indicators from other sustainability fields, i.e., each sustainability aspect receives a small group of indicators to represent such aspect.

Although there are many studies about local-sphere sustainability indicators, the topic "solid waste" in the context of these systems is little explored. Given the complexity of this topic, it is important questioning whether indicators encompassing the LSA systems can capture and showing the reality of such matter in different territories, where this topic embodies relevance for sustainability. Accordingly, the aim of the present article is to carry out a systematic literature review to understand the main features and methodological approaches applied to solid wastes in the context of local sustainability assessment systems.

\section{Solid wastes: complexity and sustainability assessment}

As for the scope of the present study, it was understood that it is essential considering the whole solid-waste context within a given location to assess local sustainability. It is important highlighting that Urban Solid Waste (USW) is only one of the generated waste types and it can be added with waste coming from other generating sources, such as healthcare services, agricultural activities, civil construction, mining, industry and any other sources found in the assessed location. Waste composition changes, not only between cities, but also within a single city throughout time. Waste features tend to seasonal variations in the short-term, and it can change in terms of amount and composition throughout the year (VERGARA; TCHOBANOGLOUS, 2012). Chart 1 presents the classification based on the generating sources and solid waste types that can be more often generated in a given location. Waste generation and allocation diversity are influenced by urbanization factors such as society's consumption income, culture, and pattern (UNITED NATIONS ENVIRONMENTAL PROGRAMME, 2010, 2011; KAZA et al., 2018).

If one takes into consideration only these two SW generation and variability features, it is possible observing that there are multiple aspects involved in their generation, namely: a) using natural resources; b) degrading natural resources, since waste is generated in locations presenting pollution loads of the most diverse natures (infecting, toxic 
and radioactive loads, among others) (MARTINEZ-ALIER, 2001); c) environmental justice, whose society unequally and unfairly press the ecosystems (developed countries vs. developing countries, North vs. South, urban population vs. rural population, among others) (WARLENIUS; PIERCE; RAMASAR, 2015); d) intergenerational equity due to waste production, consumption and, consequently, generation influencing the ability of ecosystems in keeping or broadening the opportunity to future generations (PELLETIER, 2010).

Chart 1 - Classification, generating sources and solid waste variety

\begin{tabular}{|c|c|c|c|}
\hline $\begin{array}{l}\text { Classification } \\
\text { of solid wastes }\end{array}$ & $\begin{array}{l}\text { Activity and loca- } \\
\text { tion where the } \\
\text { waste is generated }\end{array}$ & Waste types & Authors \\
\hline Domestic waste & Residences & \multirow{3}{*}{$\begin{array}{l}\text { Food leftovers, textile, } \\
\text { newspapers, packages } \\
\text { (paper, card paper, plas- } \\
\text { tic, wood, glass, cans, } \\
\text { among others), toilet } \\
\text { paper, diapers; garden- } \\
\text { ing, electronic, furni- } \\
\text { ture, tire and oil waste. }\end{array}$} & \multirow{3}{*}{$\begin{array}{l}\text { Tchobanoglous; } \\
\text { Kreith (2002); } \\
\text { Pitchel (2005); } \\
\text { Rhyner et al. } \\
\text { (1995); Williams } \\
\text { (2005); Schalch } \\
\text { et al. (2002). }\end{array}$} \\
\hline $\begin{array}{l}\text { Commercial } \\
\text { establishments } \\
\text { and service } \\
\text { provider waste }\end{array}$ & $\begin{array}{l}\text { Shops, restaurants, } \\
\text { offices, bars, super- } \\
\text { market, hotels }\end{array}$ & & \\
\hline $\begin{array}{l}\text { Institutional } \\
\text { waste }\end{array}$ & $\begin{array}{l}\text { Schools, prisons, } \\
\text { hospitals (except for } \\
\text { healthcare services) }\end{array}$ & & \\
\hline $\begin{array}{c}\text { Urban cleaning } \\
\text { service waste }\end{array}$ & $\begin{array}{l}\text { Public roads, beaches } \\
\text { and veterinary cleaning, } \\
\text { cleaning of galleries, } \\
\text { brooks, terrains, land- } \\
\text { scaping, among others }\end{array}$ & $\begin{array}{l}\text { Special residues, street } \\
\text { sweeping, tree pruning } \\
\text { leftovers, construc- } \\
\text { tion, and demolition } \\
\text { leftovers, abandoned } \\
\text { cars, animal carcasses, } \\
\text { among others. }\end{array}$ & $\begin{array}{l}\text { Tchobanoglous; } \\
\text { Kreith; Williams } \\
\text { (2002); Pitchel } \\
\text { (2005); Williams } \\
\text { (2005); Schalch } \\
\text { et al. (2002). }\end{array}$ \\
\hline $\begin{array}{c}\text { Healthcare } \\
\text { services waste }\end{array}$ & $\begin{array}{c}\text { Hospitals, medical } \\
\text { and veterinary clin- } \\
\text { ics, clinical analysis } \\
\text { laboratories, drugstores, } \\
\text { healthcare units, } \\
\text { dentistry offices and } \\
\text { other establishments }\end{array}$ & $\begin{array}{l}\text { Blood, body fluids, } \\
\text { body parts or organs of } \\
\text { humans and animals, } \\
\text { cotton, syringes, blades, } \\
\text { handling materi- } \\
\text { als, among others. }\end{array}$ & $\begin{array}{l}\text { Pitchel }(2005) ; \\
\text { Schalch et } \\
\text { al. }(2002)\end{array}$ \\
\hline $\begin{array}{l}\text { Construction } \\
\text { and demoli- } \\
\text { tion residues }\end{array}$ & $\begin{array}{l}\text { Construction sites, } \\
\text { road repair sites, } \\
\text { building demolition }\end{array}$ & $\begin{array}{l}\text { Demolition materi- } \\
\text { als, construction site } \\
\text { debris, renovation sites, } \\
\text { wood, steel, concrete, } \\
\text { rocks, dust, soil com- } \\
\text { posites, among others. }\end{array}$ & $\begin{array}{l}\text { Tchobanoglous; } \\
\text { Kreith; Williams } \\
(2002) \text {; Pitchel } \\
(2005) \text {; Williams } \\
\text { (2005); Schalch } \\
\text { et al. }(2002) \text {. }\end{array}$ \\
\hline
\end{tabular}




\begin{tabular}{|c|c|c|c|}
\hline $\begin{array}{l}\text { Transporta- } \\
\text { tion service }\end{array}$ & $\begin{array}{l}\text { Harbors, airports and } \\
\text { train and bus stations }\end{array}$ & $\begin{array}{l}\text { Hygiene materials } \\
\text { and food leftovers, } \\
\text { which can bring dis- } \\
\text { eases from other cities, } \\
\text { states, and countries. }\end{array}$ & $\begin{array}{l}\text { Pitchel (2005); } \\
\text { Schalch et } \\
\text { al. (2002). }\end{array}$ \\
\hline Industrial Waste & $\begin{array}{l}\text { Contractor companies, } \\
\text { light and heavy machin- } \\
\text { ery companies, refiner- } \\
\text { ies, chemical companies, } \\
\text { demolition compa- } \\
\text { nies, among others. }\end{array}$ & $\begin{array}{l}\text { Scarp, combustion } \\
\text { ash, foundry sand, oils, } \\
\text { silt, plastics, paper, } \\
\text { wood, fibers, food } \\
\text { waste, tires, construc- } \\
\text { tion and demolition } \\
\text { waste, special waste, } \\
\text { and hazardous waste }\end{array}$ & $\begin{array}{l}\text { Tchobanoglous; } \\
\text { Kreith; Williams } \\
\text { (2002); Pitchel } \\
\text { (2005); Rhyner et } \\
\text { al. (1995); Wil- } \\
\text { liams (2005). }\end{array}$ \\
\hline Agricultural waste & $\begin{array}{l}\text { Fields and harvest areas, } \\
\text { orchards, vineyards, } \\
\text { dairy production, farms }\end{array}$ & $\begin{array}{l}\text { Food leftovers, bio- } \\
\text { mass, and packages }\end{array}$ & $\begin{array}{l}\text { Tchobanoglous; } \\
\text { Kreith; Williams } \\
\text { (2002); Pitchel } \\
\text { (2005); Rhyner et } \\
\text { al. (1995); Wil- } \\
\text { liams (2005). }\end{array}$ \\
\hline Mining waste & Mining companies & $\begin{array}{l}\text { Soils, rocks, and left- } \\
\text { overs generated through } \\
\text { mineral and iron pro- } \\
\text { cessing }\end{array}$ & $\begin{array}{l}\text { Pitchel (2005); } \\
\text { Rhyner et al. } \\
\text { (1995); Williams } \\
\text { (2005); Bagchi } \\
\text { (2004). }\end{array}$ \\
\hline $\begin{array}{l}\text { Dangerous uni- } \\
\text { versal wastes }\end{array}$ & $\begin{array}{l}\text { Small and large compa- } \\
\text { nies, residences }\end{array}$ & $\begin{array}{l}\text { Nickel-cadmium and } \\
\text { lead acid batteries } \\
\text { (found in electronic } \\
\text { devices, mobiles, and } \\
\text { notebooks) }\end{array}$ & Pitchel (2005). \\
\hline $\begin{array}{l}\text { Nuclear process } \\
\text { wastes }\end{array}$ & $\begin{array}{l}\text { Nuclear Power Plants, } \\
\text { nuclear waste repro- } \\
\text { cessing facilities, nuke } \\
\text { facilities, research, and } \\
\text { medical procedures }\end{array}$ & $\begin{array}{l}\text { Byproducts of nuclear } \\
\text { fusion processes or } \\
\text { nuclear combustion } \\
\text { processing presenting } \\
\text { Uranium and Plutonium }\end{array}$ & Pitchel (2005). \\
\hline
\end{tabular}

Source: elaborated by the authors

Other political, normative, managerial, and operational factors interact with SW generation in its integrated management processes. Based on the current article, these factors are related to segregation processes in SW source, collection, destination (solid waste transportation, screening, processing and transformation) and final disposal (VERGARA; TCHOBANOGLOUS, 2012; WILSON et al., 2012; KAZA et al., 2018). All these processes are intrinsically related to sustainability aspects, such as: a) pollution prevention in waste final disposal or treatment focused on maintaining natural resources of the socio-ecological system (PELLETIER et al., 2014); b) adaptation to circular 
economy based on a paradigm of cycle closing, in which waste is seen as raw-material or energetic source (IACOVIDOU et al., 2017; SAVINI, 2019); c) financial efficiency in order to reduce mass and energy consumption, and cleaner production; d) interference in human well-being, since SW has the potential to generate bad smell, visual pollution, as well as to increase the proliferation of disease and contamination vectors in humans (ZHANG; MATSUTO, 2013; SIMSEK et al., 2014); e) the socio-environmental civility, and democratic and participatory socio-environmental governance, based on the source and reuse segregation, and segregation perspective about other solid waste management (SWM) stages that concern the stimuli to citizen commitment and sensitivity (THORGENSEN, 2005; BARR; GILG; SHAW, 2011; GRUNERT; ROUSTA; DAHLÉN, 2015) and to collective responsibility (GIBSON; HASSAN; TANSEY, 2005; HENRIKSSON; ÅKESSON; EWERT, 2010).

Besides, locations can present specific aspects that have impact on local sustainability. Developing countries, for example, show reports about the presence of people and animals living in dumps (BJERKLI, 2015; SAMSON, 2017), as well as about discussions about scavengers' participation in selective collections in counties, since they need to be integrated to, and valorized by, solid waste management practices (CETRULO et al., 2018; VALLIN; GONÇALVES-DIAS, 2019). On the other hand, when it comes to developed countries, locations can face issues such as high solid waste generation, mainly of electronic waste, and socio-technical dependence (locked in) on incineration plants to treat municipal solid waste (CORVELLEC; CAMPOS; ZAPATA, 2013).

However, inserting the SW dimension in local sustainability valuations by using indicator systems is not easy. Important aspects to be considered in sustainability assessment analysis are turned into indicators and incorporated to the system, and it often results in complex and dense tools. Indicator systems ended up neglecting some important points for the sustainable development of the assessed location as an attempt to holistically look to sustainable aspects. In other words, indicators can become shallow to cover most sustainability aspects and important information can be lost throughout the process. There is great probability of it to happen, in case of complex aspects, such as the solid waste topic.

Another essential matter is linked to the fact that SW complexity changes from location to location. From the sustainability assessment viewpoint, in order to apply the practical use of these systems, it is important highlighting the need of understanding the political, institutional and cultural contexts in which the indicator has been built in as a way to reflect local specifications (RYDIN; HOLMAN; WOLF, 2003; MICKWITZ; MELANEN, 2009; POLIDO; JOÃO; RAMOS, 2014). This process requires the involvement of communities affected by these indicators; therefore, it is necessary building such indicators in compliance with reality (MORENO-PIRES, 2011; SINGH et al., 2012). It is certain that factors relevant to a given location will be assessed through the indicator system after communities get involved in indicator construction; in other words, indicators will be chosen based on what must be evaluated in the community in question (BELL; MORSE, 2001; FRASER et al., 2006). The community involvement is of paramount 
importance for the solid waste matter, since this issue is part of individuals' daily lives and starts in the very core of residences and households (BULKELEY; GREGSON, 2009; RØPKE, 2009; BARR; GILG; SHAW, 2011). Therefore, this aspect reflects the degree of society's involvement in the solid waste issue, and it depends on including people in SW management and on improving sustainability-related factors, because only these individuals understand and feel local issues caused by waste. Besides, social actors' participation in the process to generate indicators is essential to consolidate a sustainability assessment tool and to reinforce the aspects of stimuli given to socio-environmental civility and democratic governance (MEADOWS, 1998; VALENTIN; SPANGENBERG, 2000; RAMOS; CAEIRO; MELO, 2004; REED; FRASER; DOUGILL, 2006). Thus, the concept of governance gained significant importance because it embodied political determinants linked to many interests and to the reality of social actors involved in SW management, in each location.

\section{Method}

The systematic literature review was carried out in scientific articles published in indexed journals that approach indicator systems (IS) in local sustainability assessment contexts. The research used Scopus ${ }^{\circledR}$ database because this is the biggest database of peer review literature. The search parameters were articles and reviews published in the last 15 years (from 2002 to May 2017). The following search meshes were used: "sustainability indicators" and "cities"; "sustainability indicators" and "local"; "sustainable development indicators" and "cities"; "sustainable development indicators" and "local"; "solid waste indicators"; "solid waste" and "sustainability indicators"; "solid waste" and "sustainable development indicators". Articles that did not present solid waste indicators in sustainability assessment tools and the ones that did not regard the "local context" (cities, regions, counties or communities) were excluded from the group of 651 articles found after the search.

In total, 77 articles were selected through data analysis, as recommended by Krippendorf (2004) and Fereday and Muir-Cochrane (2006). The process concerned identifying these subjects through reading, second reading and information analysis. Accordingly, it was possible valuing patterns and the three groups of articles found through the search: a) the first group was composed of ISs that assess the technical performance of solid waste management ( 25 articles) and approached subjects such as material flow analysis (ZACCARIELLO; CREMIATO; MASTELLONE, 2015), energy recovery rate (BUENO; LATASA; LOZANO, 2015), techniques to evaluate and improve the performance of recollection, treatment and management systems (ADAMOVIĆ et al., 2017; CHAMIZO-GONZALEZ; CANO-MONTERO; MUÑOZ-COLOMINA, 2016; PLATADÍAZ et al., 2014), biological treatments (LOMBARDI; CARNEVALE; CORTI, 2015) and innovations (NING; CHANG; HUNG, 2013). Therefore, indicators encompassing these systems are not dedicated to local sustainability assessment; b) ISs, in the second group, were exclusively dedicated to the SW component (VEIGA et al., 2016; CASTRO; SILVA; MARCHAND, 2015; SANTIAGO; DIAS, 2012), although the focus lied on 
the assessment of solid waste sustainability management rather than on location sustainability assessment (16 studies); c) the last group was composed of indicators that were inserted in IS to evaluate local sustainability (36 articles) and solid waste by adding to the sustainability aspects that compose the tools. Only articles in this last category were included in the present review.

Three analysis stages were carried out with 36 articles included in the systematic review. The first stage described study features (quantitative analysis) based on journal, publication field, number of authors, temporal and spatial distribution of studies (Section 4).

The second stage focused on the construction of the local sustainability assessment system process and on the representativeness of solid waste indicators in local sustainability assessment systems in order to understand how the insertion of the solid waste subject took place in Indicator Systems. Therefore, the following factors were assessed: the total number of indicator systems and SW indicators, the IS organization adopted by the author (sustainable development dimensions or thematic areas), dimensions of allocated SW indicators and the process to construct the local sustainability assessment system (Section 5).

Finally, an analysis was carried out (Section 6) to assess whether the chosen indicators composing IS, and to represent SW complexity and its association with sustainability. Therefore, all SW indicators were classified based on:

a) The stage process that indicator represents in SWM: generation, collection, destination, final disposal or governance [this division was adopted by taking into account concepts presented by Tchobanoglous, Kreith, and Williams (2002), Vergara and Tchobanoglous (2012) and by the European Guideline 2008/98/EC;

b) The importance level represented by indicator in SWM hierarchy (based on the waste hierarchy in Directive 2008/98/EC due to its consistency with sustainability aspects. The strategic priority lies on the non-generation of solids, which is followed by waste reuse, recycling and recovery actions until final disposal);

c) Indicators has potential to represent sustainability aspects (systematized in environmental, economic, and social dimensions).

\section{Study features}

The chronological analysis of the 36 selected articles for the literature review pointed towards a larger number of publications on the herein addressed subject in the last 15 years (Figure 1), with emphasis on 2010 and 2015. 
Figure 1 - Distribution of scientific articles on local sustainability assessment that have counted on SW indicators, per publication year.

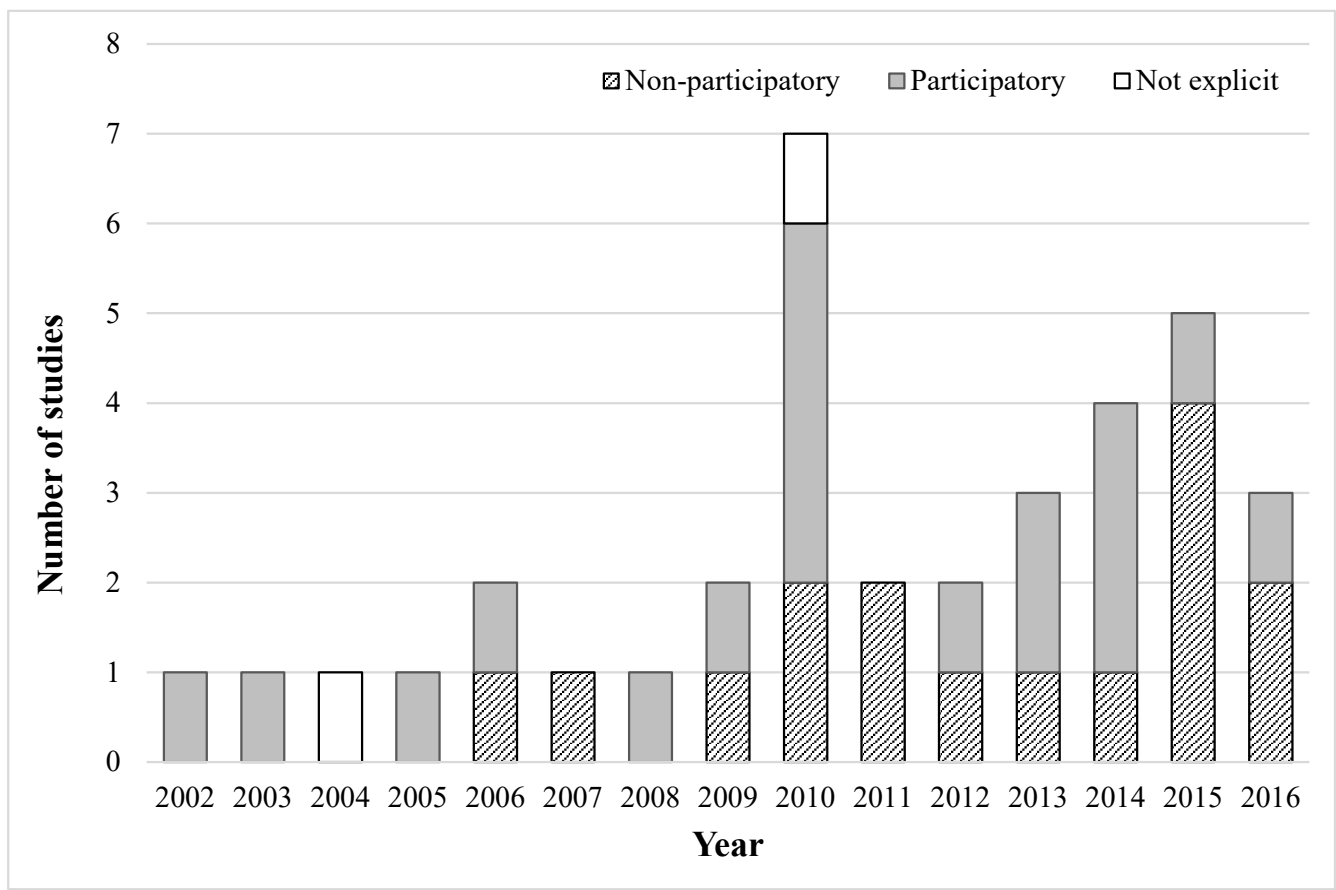

Source: elaborated by the author

Sustainability, initially explored in agreements depicted at international scale, was added to policies, programs, and projects at local scale (ICLEI, 2017; MAPAR et al., 2017). Evidence of this new guideline were incorporated to ODS 11 (UNITED NATION, 2015), which addresses Sustainable Cities and Communities. The importance of a given location in the search for sustainable development point towards the need of adopting tools to evaluate local sustainability (MAPAR et al., 2017). Similarly, this discussion broadened in the literature about the analysis of consolidated indicator systems (SHEN et al., 2011; KING, 2016), as well as about new propositions and outcomes concerning this subject at local scale (MASCARENHAS; NUNES; RAMOS, 2015) - its role in tool development is better discussed in Section 5.

With respect to publication field, most articles were published in Environmental Sciences field journals (14\%). There are published studies in the Energy (11\%), Social Sciences (8\%) and Engineering (5\%) fields, among others, although with lower representativeness. This diversity in study publication fields can be explained by the multi-dimensional and interdisciplinary features of sustainability, which is even more significant for assessment tools (BOSSEL, 1999; RAMOS; CAEIRO, 2010). On the other hand, Environmental Sciences present a broad scope by incorporating associations between environment and society, providing a more holistic profile to the study object. Accordingly, there are publication indexation to local sustainability assessment issues. 
The literature review pointed out significant dispersion between journals that have already published articles on this topic, mainly in the journal Ecological Indicators (7 articles), which was followed by the journal Sustainability (4 articles). The list of journals, as well as the respective number of articles about SW indicators inserted in systems to evaluate local sustainability are shown in Chart 2.

\section{Chart 2 - Distribution of scientific articles to evaluate local sus- tainability focused on SW indicators, per journal}

\begin{tabular}{|c|c|}
\hline Journal & $\begin{array}{c}\text { Number of } \\
\text { Studies }\end{array}$ \\
\hline Ecological indicators & 7 \\
\hline Sustainability & 4 \\
\hline Cities & 2 \\
\hline Environmental Impact Assessment Review & 2 \\
\hline Journal of Environmental Planning and Management & 2 \\
\hline Tourism Management & 2 \\
\hline Journal of Environmental Management & 2 \\
\hline Australian Journal of Social Issues & 1 \\
\hline Building and Environment & 1 \\
\hline Environment, Development and Sustainability & 1 \\
\hline Environmental Progress \& Sustainable Energy & 1 \\
\hline European Planning Studies & 1 \\
\hline Habitat International & 1 \\
\hline Int. J. Sustainable Society & 1 \\
\hline International Journal of Sustainable Development \& World Ecology & 1 \\
\hline International review for spatial planning and sustainable development & 1 \\
\hline Journal of Cleaner Production & 1 \\
\hline Local Environment & 1 \\
\hline Progress in Planning & 1 \\
\hline Rev. Ambient. Água & 1 \\
\hline Sustainability Science & 1 \\
\hline URBE - Brazilian Journal of Urban Management & 1 \\
\hline Total & 36 \\
\hline
\end{tabular}

Source: elaborated by the author

It is important highlighting that most selected studies (89\%) were produced by more than one author. These data may have been mostly influenced by semantic and interdisciplinary features of discussions about sustainability assessment. In total, 99 different authors were identified among the selected articles, 5 of them were collaborators 
in two references, and only one author was collaborator in three articles.

With respect to authors' origin, 47\% were European, with emphasis on Portugal ( 8 researchers who addressed the subject). Among the $24 \%$ of Asian and the $22 \%$ of American authors, 8 were Chinese; Brazil stood out for its 11 authors. There was also the participation of researchers from Oceania since they accounted for $7 \%$ of the total of authors. European locations in an analysis grouped by continent were assessed in more than $30 \%$ of the studies (notice that almost half of authors identified in the present review were European), 22\% of the articles focused on the analysis of locations in America, 14\% focused on Asia and $8 \%$ on Oceania. The absence of authors and IS of African Continent was noteworthy.

\section{Features of indicator systems}

This section focuses on exploring how the solid waste subject was inserted in Indicator Systems. As it can be observed in Chart 3, these systems are developed at local level, either for communities, cities, or regions. There is great variability in IS organization or in the type and number of categories. For example, Nader, Salloum, and Karam (2008) divided the system into four topics; Castellani and Sala (2010) used six fields; Fehr et al. (2004) used twelve sustainability parameters; and Braulio-Gonzalo, Bovea, and Ruá (2015) used 14 categories.

Chart 3: Features of local sustainability assessment indicator systems, which count on SW indicators.

\begin{tabular}{|c|c|c|c|c|c|}
\hline Source & Region & IS organization & $\begin{array}{c}\text { Number } \\
\text { of indi- } \\
\text { cators }\end{array}$ & $\begin{array}{c}\text { Number } \\
\text { of SW } \\
\text { indicators }\end{array}$ & $\begin{array}{c}\text { Process to } \\
\text { construct } \\
\text { the IS }\end{array}$ \\
\hline $\begin{array}{c}\text { Kelly and } \\
\text { Moles } \\
\text { (2002) }\end{array}$ & $\begin{array}{c}\text { Ireland } \\
\text { cities }\end{array}$ & $\begin{array}{c}\text { Environmental; So- } \\
\text { cial; Economic }\end{array}$ & 25 & 2 & Participatory \\
\hline $\begin{array}{c}\text { Yuan et } \\
\text { al. (2003) }\end{array}$ & $\begin{array}{c}\text { City } \\
\text { (Shang- } \\
\text { hai, } \\
\text { China) }\end{array}$ & Without categorization structure & 17 & 1 & Participatory \\
\hline $\begin{array}{c}\text { Fehr et al. } \\
\text { (2004) }\end{array}$ & $\begin{array}{c}\text { Cities } \\
\text { (Brazil) }\end{array}$ & $\begin{array}{c}\text { Demographic density and } \\
\text { Evolution; Public transportation; } \\
\text { Solid waste handling; Liquid } \\
\text { effluent handling; Air monitor- } \\
\text { ing; Fresh water supply; Public } \\
\text { education; Public health care; } \\
\text { Cultural manifestations; Energy } \\
\text { supply; Park maintenance; Land } \\
\text { use and resource preservation }\end{array}$ & 68 & 6 & Not explicit \\
\hline
\end{tabular}




\begin{tabular}{|c|c|c|c|c|c|}
\hline $\begin{array}{l}\text { McAlpine } \\
\text { and Birnie } \\
(2005)\end{array}$ & $\begin{array}{l}\text { Island of } \\
\text { Guernsey }\end{array}$ & Without categorization structure & 55 & 3 & Participatory \\
\hline $\begin{array}{c}\text { Choi and } \\
\text { Sirakaya } \\
(2006)\end{array}$ & $\begin{array}{l}\text { Com- } \\
\text { munity }\end{array}$ & $\begin{array}{c}\text { Social; Economic; Ecological; } \\
\text { Political; Technological; Cultural }\end{array}$ & 125 & 3 & $\begin{array}{l}\text { Non-par- } \\
\text { ticipatory }\end{array}$ \\
\hline $\begin{array}{l}\text { Fraser et } \\
\text { al. }(2006)\end{array}$ & $\begin{array}{l}\text { Island of } \\
\text { Guernsey }\end{array}$ & Without categorization structure & 17 & 3 & Participatory \\
\hline $\begin{array}{c}\text { Lee and } \\
\text { Huang } \\
(2007)\end{array}$ & $\begin{array}{l}\text { Taipei } \\
\text { City } \\
\text { (Taiwan) } \\
\end{array}$ & $\begin{array}{l}\text { Environmental; Social; } \\
\text { Economic; Institutional }\end{array}$ & 51 & 3 & $\begin{array}{l}\text { Non-par- } \\
\text { ticipatory }\end{array}$ \\
\hline $\begin{array}{l}\text { Nader, } \\
\text { Salloum } \\
\text { and } \\
\text { Karam } \\
(2008)\end{array}$ & $\begin{array}{l}\text { Lebanon } \\
\text { munici- } \\
\text { palities }\end{array}$ & $\begin{array}{l}\text { Population and socio-economic } \\
\text { indicators; Economic indica- } \\
\text { tors; Environment indicators; } \\
\text { Sustainable development activi- } \\
\text { ties and policies indicators }\end{array}$ & 110 & 6 & Participatory \\
\hline $\begin{array}{l}\text { Mick- } \\
\text { witz and } \\
\text { Melanen } \\
(2009)\end{array}$ & $\begin{array}{l}\text { Kymen- } \\
\text { laakso } \\
\text { Region } \\
\text { (Finland) }\end{array}$ & Without categorization structure & 50 & 2 & Participatory \\
\hline $\begin{array}{l}\text { Spilanis et } \\
\text { al. (2009) }\end{array}$ & $\begin{array}{l}\text { Islands } \\
\text { of Paros } \\
\text { and Kos } \\
\text { (Greece) }\end{array}$ & $\begin{array}{l}\text { Environmental; So- } \\
\text { cial; Economic. }\end{array}$ & 39 & 1 & $\begin{array}{l}\text { Non-par- } \\
\text { ticipatory }\end{array}$ \\
\hline $\begin{array}{l}\text { Castellani } \\
\text { and Sala } \\
(2010)\end{array}$ & $\begin{array}{l}\text { Com- } \\
\text { munity }\end{array}$ & $\begin{array}{l}\text { Population; Housing; Ser- } \\
\text { vices; Economy and labour; } \\
\text { Environment; Tourism }\end{array}$ & 20 & 1 & Participatory \\
\hline $\begin{array}{l}\text { Chávez- } \\
\text { Cortés } \\
\text { and Maya } \\
(2010)\end{array}$ & $\begin{array}{l}\text { Com- } \\
\text { munity }\end{array}$ & $\begin{array}{l}\text { Environmental; Social; } \\
\text { Economic; Institutional }\end{array}$ & 34 & 2 & Participatory \\
\hline $\begin{array}{l}\text { Cox et al. } \\
(2010)\end{array}$ & $\begin{array}{l}\text { Com- } \\
\text { munity } \\
\text { (Victoria, } \\
\text { Australia) }\end{array}$ & $\begin{array}{l}\text { Healthy, safe and inclusive } \\
\text { communities; Dynamic, resilient } \\
\text { local economies; Sustainable } \\
\text { built and natural environments; } \\
\text { Culturally rich and vibrant } \\
\text { communities; Democratic } \\
\text { and engaged communities }\end{array}$ & 90 & 2 & Participatory \\
\hline $\begin{array}{l}\text { Mascar- } \\
\text { enhas et } \\
\text { al. }(2010)\end{array}$ & $\begin{array}{c}\text { Algarve } \\
\text { (Portugal) }\end{array}$ & Without categorization structure & 20 & 2 & Participatory \\
\hline $\begin{array}{l}\text { Tanguay } \\
\text { et al. } \\
(2010)\end{array}$ & $\begin{array}{l}\text { Cities } \\
\text { (US, Can- } \\
\text { ada, and } \\
\text { Europe) }\end{array}$ & $\begin{array}{l}\text { Environmental; So- } \\
\quad \text { cial; Economic; } \\
\text { Livable; Equitable; Vi- } \\
\text { able; Sustainable. }\end{array}$ & 29 & 2 & $\begin{array}{l}\text { Non-par- } \\
\text { ticipatory }\end{array}$ \\
\hline
\end{tabular}




\begin{tabular}{|c|c|c|c|c|c|}
\hline $\begin{array}{l}\text { Tolón- } \\
\text { Becerra, } \\
\text { Lastra- } \\
\text { Bravo and } \\
\text { Galdeano- } \\
\text { Gómez } \\
(2010)\end{array}$ & $\begin{array}{l}\text { Commu- } \\
\text { nity (An- } \\
\text { dalusia- } \\
\text { Spanish) }\end{array}$ & $\begin{array}{l}\text { Labor means; Demography; } \\
\text { Local economy; Tourism, } \\
\text { Energy and Transport; Ag- } \\
\text { riculture and Livestock; } \\
\text { Natural resources; so- } \\
\text { cial resources }\end{array}$ & 228 & 2 & Not explicit \\
\hline $\begin{array}{l}\text { Yigitcanlar } \\
\text { and Dur } \\
(2010)\end{array}$ & $\begin{array}{l}\text { City (Gold } \\
\text { Coast, } \\
\text { Australia) }\end{array}$ & $\begin{array}{l}\text { Environmet; Demography; Land } \\
\text { use and urban form; Transport }\end{array}$ & 29 & 1 & $\begin{array}{l}\text { Non-par- } \\
\text { ticipatory }\end{array}$ \\
\hline $\begin{array}{l}\text { Choon et } \\
\text { al. }(2011)\end{array}$ & $\begin{array}{c}\text { Cities } \\
\text { (Malaysia) }\end{array}$ & $\begin{array}{c}\text { Environmental well-being; } \\
\text { Human well-being }\end{array}$ & 30 & 5 & $\begin{array}{l}\text { Non-par- } \\
\text { ticipatory }\end{array}$ \\
\hline $\begin{array}{l}\text { Shen et } \\
\text { al. }(2011)\end{array}$ & $\begin{array}{c}\text { Cities } \\
\text { (Asia, } \\
\text { Europe, } \\
\text { and North } \\
\text { America) }\end{array}$ & $\begin{array}{l}\text { Environmental; Social; } \\
\text { Economic; Governance }\end{array}$ & 37 & 1 & $\begin{array}{l}\text { Non-par- } \\
\text { ticipatory }\end{array}$ \\
\hline $\begin{array}{l}\text { Turcu } \\
(2012)\end{array}$ & $\begin{array}{l}\text { Com- } \\
\text { munity } \\
\text { (English } \\
\text { neigh- } \\
\text { bour- } \\
\text { hoods) }\end{array}$ & $\begin{array}{l}\text { Environmental sustain- } \\
\text { ability; Social sustainabil- } \\
\text { ity; Economic sustainability; } \\
\text { Institutional sustainability }\end{array}$ & 26 & 1 & Participatory \\
\hline $\begin{array}{l}\text { Xu and } \\
\text { Coors } \\
(2012)\end{array}$ & $\begin{array}{l}\text { Commu- } \\
\text { nity (Ger- } \\
\text { many) }\end{array}$ & $\begin{array}{l}\text { Environment; Hous- } \\
\text { ing; Economics; Society }\end{array}$ & 24 & 1 & $\begin{array}{l}\text { Non-par- } \\
\text { ticipatory }\end{array}$ \\
\hline $\begin{array}{l}\text { Castellani } \\
\text { and Sala } \\
(2013)\end{array}$ & $\begin{array}{l}\text { Cities } \\
\text { (Italy) }\end{array}$ & $\begin{array}{l}\text { Ar and climate; Water; Land } \\
\text { use; Agriculture; Biodiversity } \\
\text { and landscape; Waste; Energy }\end{array}$ & 21 & 2 & $\begin{array}{l}\text { Non-par- } \\
\text { ticipatory }\end{array}$ \\
\hline $\begin{array}{l}\text { Tourneau } \\
\text { et al. } \\
(2013)\end{array}$ & $\begin{array}{l}\text { Com- } \\
\text { munity } \\
\text { (Brazil) }\end{array}$ & $\begin{array}{l}\text { Life conditions; Environmental } \\
\text { conditions; Present } \\
\text { needs and future } \\
\text { perspective; Governance }\end{array}$ & 44 & 1 & Participatory \\
\hline $\begin{array}{l}\text { Turcu } \\
(2013)\end{array}$ & $\begin{array}{l}\text { Com- } \\
\text { munity } \\
\text { (United } \\
\text { Kingdom) }\end{array}$ & $\begin{array}{l}\text { Environmental sustain- } \\
\text { ability; Social sustainabil- } \\
\text { ity; Economic sustainability; } \\
\text { Institutional sustainability }\end{array}$ & 24 & 1 & Participatory \\
\hline
\end{tabular}




\begin{tabular}{|c|c|c|c|c|c|}
\hline $\begin{array}{l}\text { Canavese, } \\
\text { Ortega } \\
\text { and } \\
\text { Queirós } \\
(2014)\end{array}$ & $\begin{array}{l}\text { Algarve } \\
\text { (Portugal) }\end{array}$ & $\begin{array}{c}\text { Demography; Health; } \\
\text { Water utilization } \\
\text { and quality; Waste production } \\
\text { and treatment; } \\
\text { Air pollution; Financing }\end{array}$ & 13 & 2 & $\begin{array}{l}\text { Non-par- } \\
\text { ticipatory }\end{array}$ \\
\hline $\begin{array}{l}\text { Graymore } \\
(2014)\end{array}$ & $\begin{array}{l}\text { Com- } \\
\text { munity } \\
\text { (Victoria, } \\
\text { Australia) }\end{array}$ & $\begin{array}{l}\text { Environmental well-being; } \\
\text { Human well-being }\end{array}$ & 41 & 2 & Participatory \\
\hline $\begin{array}{c}\text { Mascar- } \\
\text { enhas, } \\
\text { Nunes } \\
\text { and } \\
\text { Ramos } \\
\text { (2014) }\end{array}$ & $\begin{array}{l}\text { Algarve } \\
\text { (Portugal) }\end{array}$ & Without categorization structure & 14 & 1 & Participatory \\
\hline $\begin{array}{l}\text { Moreno } \\
\text { Pires, } \\
\text { Fidélis and } \\
\text { Ramos } \\
\text { (2014) }\end{array}$ & $\begin{array}{c}\text { Cities } \\
\text { (Cascais } \\
\text { and } \\
\text { Oeiras, } \\
\text { Portugal) }\end{array}$ & $\begin{array}{c}\text { Sustainable } \\
\text { Development Educa- } \\
\text { tion; Marine and } \\
\text { Coastal Environment Institu- } \\
\text { tions; Nature Conservation and } \\
\text { Biodiversity; Nature Conserva- } \\
\text { tion, Forest Planning; Air; Water; } \\
\text { Waste; Energy; Transport; Noise; } \\
\text { Agriculture; Tourism }\end{array}$ & 21 & 1 & Participatory \\
\hline $\begin{array}{l}\text { Braulio- } \\
\text { Gonzalo, } \\
\text { Bovea } \\
\text { and Ruá } \\
(2015)\end{array}$ & $\begin{array}{l}\text { City } \\
\text { (Spain) }\end{array}$ & $\begin{array}{l}\text { Site and soil; Urban morphol- } \\
\text { ogy; Mobility and transport; } \\
\text { Nature and biodiversity; Building } \\
\text { and housing; Energy; Water } \\
\text { Materials; Waste; Pollu- } \\
\text { tion; Social aspect; Economic } \\
\text { aspect; Management and } \\
\text { institution; Innovation }\end{array}$ & 63 & 2 & $\begin{array}{l}\text { Non-par- } \\
\text { ticipatory }\end{array}$ \\
\hline $\begin{array}{l}\text { Egilmez, } \\
\text { Gumus } \\
\text { and } \\
\text { Kucukvar } \\
(2015)\end{array}$ & $\begin{array}{l}\text { Cities } \\
\text { (US and } \\
\text { Canada) }\end{array}$ & $\begin{array}{c}\text { Air; Energy use; Build- } \\
\text { ings; Water; } \\
\text { Waste; Land use; Transport }\end{array}$ & 16 & 1 & $\begin{array}{l}\text { Non-par- } \\
\text { ticipatory }\end{array}$ \\
\hline $\begin{array}{l}\text { Martins } \\
\text { and } \\
\text { Cândido } \\
\text { (2015) }\end{array}$ & $\begin{array}{l}\text { Cities } \\
\text { (Brazil) }\end{array}$ & $\begin{array}{c}\text { Eco-energetic rationality; Urban } \\
\text { metabolism; Purity; Citizenship; } \\
\text { Efficiency; Equity; Patrimony }\end{array}$ & 172 & 15 & $\begin{array}{l}\text { Non-par- } \\
\text { ticipatory }\end{array}$ \\
\hline
\end{tabular}




\begin{tabular}{|c|c|c|c|c|c|}
\hline $\begin{array}{l}\text { Robati, } \\
\text { Mona- } \\
\text { vari and } \\
\text { Majedi } \\
(2015) \\
\end{array}$ & $\begin{array}{l}\text { City } \\
\text { (Iran) }\end{array}$ & $\begin{array}{l}\text { Physical; Socioeco- } \\
\text { nomic; Cultural }\end{array}$ & 16 & 2 & $\begin{array}{l}\text { Non-par- } \\
\text { ticipatory }\end{array}$ \\
\hline $\begin{array}{l}\text { Sui-Qui } \\
\text { and Leng } \\
(2015)\end{array}$ & $\begin{array}{c}\text { City } \\
\text { (Mexico) }\end{array}$ & $\begin{array}{l}\text { Environment; Socio-eco- } \\
\text { nomic Environment; Urban } \\
\text { development; Tourism }\end{array}$ & 50 & 6 & Participatory \\
\hline $\begin{array}{l}\text { King } \\
(2016)\end{array}$ & $\begin{array}{l}\text { City } \\
\text { (US) }\end{array}$ & $\begin{array}{l}\text { Economic; Environ- } \\
\text { mental; Social }\end{array}$ & 25 & 1 & $\begin{array}{l}\text { Non-par- } \\
\text { ticipatory }\end{array}$ \\
\hline $\begin{array}{l}\text { Mani- } \\
\text { tiu and } \\
\text { Pedrini } \\
(2016)\end{array}$ & $\begin{array}{c}\text { Cities } \\
\text { (Europe) }\end{array}$ & $\begin{array}{l}\text { Environmental dimen- } \\
\text { sion; Social; Cultural }\end{array}$ & 61 & 2 & $\begin{array}{l}\text { Non-par- } \\
\text { ticipatory }\end{array}$ \\
\hline $\begin{array}{c}\text { Pereira } \\
\text { and Vieira } \\
(2016)\end{array}$ & $\begin{array}{l}\text { City } \\
\text { (Brazil) }\end{array}$ & Without categorization structure & 11 & 1 & $\begin{array}{l}\text { Non-par- } \\
\text { ticipatory }\end{array}$ \\
\hline
\end{tabular}

Source: Elaborated by the author

Note:

a Categories used by the authors to organize IS can vary, for example: dimensions, sectors, fields, topics, parameters. Categories including SW indicators are highlighted in bold.

There is great difference in categories SW indicators are inserted in, and it makes sense because there are several categories. However, it was noticed that a certain aspect of solid waste can be allocated in categories that present divergent purposes. For example, there is the indicator applied to hospital waste generation, which, in the system presented by Nader, Salloum and Karam (2008), is allocated in the topic "Population and socio-economic indicators" and in the IS proposed by Martins and Cândido (2015): "eco-energetic rationality".

Regarding the construction process of sustainability assessment tools, it was only possible observing Public participation in the construction of indicator systems in half of the assessed studies (Chart 3). IS built without the involvement of the interested parts was based on an approach of top down decision-making. Results did not evidence association between the participation of social actors and the amount of solid waste indicators in IS.

As for the representativeness of solid waste indicators in local sustainability assessment systems, a frequency analysis evidenced that more than $80 \%$ of ISs present only one, two or three SW indicators ( $\mathrm{fi}=15,12$ and 4, respectively). Only four studies presented five or more indicators; Martins and Cândido (2015) included 15 SW indicators.

\section{Features of solid waste indicators}

Chart 4 presents the 50 solid waste indicators found in IS for ASL and identifies 
objects they aim at representing. These objects were divided into three categories: SWM process stage, importance level at SWM hierarchy and sustainability dimensions.

Chart 4: Features of solid waste indicators found in IS, based on its potential to represent the SWM process stage, on importance level at SWM hierarchy and on sustainability dimensions.

\begin{tabular}{|c|c|c|c|c|}
\hline Indicator & Source & $\begin{array}{l}\text { State in } \\
\text { the SWM } \\
\text { process }\end{array}$ & $\begin{array}{l}\text { Level in the } \\
\text { SWM hierarchy }\end{array}$ & $\begin{array}{l}\text { Sustainability } \\
\text { dimensions }\end{array}$ \\
\hline $\begin{array}{c}\text { House- } \\
\text { hold waste } \\
\text { generation }\end{array}$ & $\begin{array}{l}\text { Tolón-Becerra, Lastra- } \\
\text { Bravo and Galdeano-Gómez } \\
\text { (2010); Cox et al. (2010); } \\
\text { Yigitcanlar and Dur (2010); } \\
\text { Robati, Monavari and } \\
\text { Majedi (2015); King (2016); } \\
\text { Shen et al. (2011); Nader, } \\
\text { Salloum and Karam (2008); } \\
\text { Sui-Qui and Leng (2015); } \\
\text { Lee and Huang (2007); } \\
\text { Castellani and Sala (2013); } \\
\text { Mcalpine and Birnie (2005); } \\
\text { Fraser et al. (2006); Xu } \\
\text { and Coors (2012); Chávez- } \\
\text { Cortés and Maya (2010); } \\
\text { Martins and Cândido (2015) }\end{array}$ & Generation & High priority & $\begin{array}{l}\text { Environmental, So- } \\
\text { cial, and Economic }\end{array}$ \\
\hline $\begin{array}{l}\text { Production } \\
\text { of Municipal } \\
\text { solid waste by } \\
\text { type of waste }\end{array}$ & Mascarenhas et al. (2010) & Generation & High priority & $\begin{array}{l}\text { Environmental, So- } \\
\text { cial, and Economic }\end{array}$ \\
\hline $\begin{array}{l}\text { Construction } \\
\text { and demoli- } \\
\text { tion waste } \\
\text { per capita }\end{array}$ & Martins and Cândido (2015) & Generation & High priority & $\begin{array}{l}\text { Environmental } \\
\text { and Economic }\end{array}$ \\
\hline $\begin{array}{c}\text { Waste per } \\
\text { capita collect- } \\
\text { ed by drop-off } \\
\text { stations }\end{array}$ & Martins and Cândido (2015) & Generation & High priority & $\begin{array}{l}\text { Environmental, So- } \\
\text { cial, and Economic }\end{array}$ \\
\hline $\begin{array}{c}\text { Amount of } \\
\text { pruning waste } \\
\text { per capita }\end{array}$ & Martins and Cândido (2015) & Generation & High priority & Environmental \\
\hline $\begin{array}{l}\text { Composition } \\
\text { of munici- } \\
\text { pal waste }\end{array}$ & $\begin{array}{l}\text { Nader, Salloum and } \\
\text { Karam (2008) }\end{array}$ & Generation & High priority & $\begin{array}{l}\text { Environmental } \\
\text { and Social }\end{array}$ \\
\hline $\begin{array}{l}\text { Hazard- } \\
\text { ous waste }\end{array}$ & $\begin{array}{l}\text { Sui-Qui and Leng (2015); } \\
\text { Kelly and Moles (2002) }\end{array}$ & $\begin{array}{l}\text { Generation } \\
\text { (Hazardous) }\end{array}$ & High priority & Environmental \\
\hline
\end{tabular}




\begin{tabular}{|c|c|c|c|c|}
\hline $\begin{array}{l}\text { Generation } \\
\text { of hospital } \\
\text { waste }^{3}\end{array}$ & $\begin{array}{l}\text { Nader, Salloum and } \\
\text { Karam (2008); Martins } \\
\text { and Cândido (2015) }\end{array}$ & $\begin{array}{c}\text { Generation } \\
\text { (Hazardous) }\end{array}$ & High priority & Environmental \\
\hline $\begin{array}{l}\text { Collected } \\
\text { solid waste } \\
\text { per inhabit- } \\
\text { ant and year }\end{array}$ & $\begin{array}{l}\text { Manitiu and Pedrini (2016); } \\
\text { Choon et al. (2013) }\end{array}$ & $\begin{array}{c}\text { Generation } \\
\text { and col- } \\
\text { lection }\end{array}$ & High priority & $\begin{array}{l}\text { Environmental } \\
\text { and Social }\end{array}$ \\
\hline $\begin{array}{l}\text { Production } \\
\text { and Selective } \\
\text { Collection } \\
\text { of Municipal } \\
\text { Waste and } \\
\text { Recovery of } \\
\text { Municipal } \\
\text { Waste }\end{array}$ & $\begin{array}{l}\text { Moreno Pires, Fidélis, } \\
\text { and Ramos (2014) }\end{array}$ & $\begin{array}{c}\text { Generation, } \\
\text { collec- } \\
\text { tion, and } \\
\text { destination }\end{array}$ & $\begin{array}{l}\text { Medium and } \\
\text { high priority }\end{array}$ & $\begin{array}{l}\text { Environmental, So- } \\
\text { cial, and Economic }\end{array}$ \\
\hline $\begin{array}{l}\text { Coverage } \\
\text { of garbage }^{\text {collection }^{1}}\end{array}$ & $\begin{array}{l}\text { Pereira and Vieira (2016); } \\
\text { Canavese, Ortega } \\
\text { and Queirós (2014); } \\
\text { Choon et al. (2013) }\end{array}$ & Collection & Low priority & $\begin{array}{l}\text { Environmental } \\
\text { and Social }\end{array}$ \\
\hline $\begin{array}{l}\text { Efficient } \\
\text { collection } \\
\text { system }^{2}\end{array}$ & $\begin{array}{l}\text { Sui-Qui and Leng (2015); } \\
\text { Martins and Cândido (2015) }\end{array}$ & Collection & Low priority & Economic \\
\hline $\begin{array}{l}\% \text { of separate } \\
\text { waste collec- } \\
\text { tion/target }\end{array}$ & Castellani and Sala (2013) & Collection & $*$ & * \\
\hline $\begin{array}{c}\text { Type of } \\
\text { collection }\end{array}$ & Fehr et al. (2014) & Collection & $*$ & $*$ \\
\hline $\begin{array}{l}\text { Proportion of } \\
\text { households } \\
\text { with proper } \\
\text { disposal } \\
\end{array}$ & Martins and Cândido (2015) & Collection & Low priority & $\begin{array}{l}\text { Environmental } \\
\text { and Social }\end{array}$ \\
\hline $\begin{array}{l}\text { Distance } \\
\text { from housing } \\
\text { to selec- } \\
\text { tive garbage } \\
\text { containers }\end{array}$ & $\begin{array}{l}\text { Braulio-Gonzalo } \\
\text { et al. (2015) }\end{array}$ & Collection & Medium priority & Social \\
\hline $\begin{array}{c}\text { Frequency of } \\
\text { collection }\end{array}$ & Fehr et al. (2014) & Collection & Low priority & $\begin{array}{c}\text { Environmental } \\
\text { and Social }\end{array}$ \\
\hline $\begin{array}{l}\text { Selective } \\
\text { waste col- } \\
\text { lection }^{4}\end{array}$ & $\begin{array}{c}\text { Martins and Cândido } \\
\text { (2015); Castellani and Sala } \\
\text { (2010); Canavese, Ortega } \\
\text { and Queirós (2014) }\end{array}$ & Collection & Medium priority & $\begin{array}{l}\text { Environmental } \\
\text { and Social }\end{array}$ \\
\hline
\end{tabular}




\begin{tabular}{|c|c|c|c|c|}
\hline $\begin{array}{c}\text { Number } \\
\text { and \% of } \\
\text { households } \\
\text { experiencing } \\
\text { problems in } \\
\text { disposing of } \\
\text { household } \\
\text { wastes }\end{array}$ & $\begin{array}{l}\text { Nader, Salloum and } \\
\text { Karam (2008) }\end{array}$ & Collection & Low priority & $\begin{array}{c}\text { Environmental } \\
\text { and Social }\end{array}$ \\
\hline $\begin{array}{l}\text { Rubbish col- } \\
\text { lection and } \\
\text { recycling cen- } \\
\text { tre ratio per } \\
10,000 \\
\text { population }\end{array}$ & Choon et al. (2013) & $\begin{array}{l}\text { Collec- } \\
\text { tion and } \\
\text { destination }\end{array}$ & $\begin{array}{l}\text { Medium and } \\
\text { low priority }\end{array}$ & $\begin{array}{l}\text { Environmental, So- } \\
\text { cial and Economic }\end{array}$ \\
\hline $\begin{array}{l}\text { Waste } \\
\text { treatment } \\
\text { and man- } \\
\text { agement }\end{array}$ & Yuan et al. (2003) & $\begin{array}{l}\text { Collection, } \\
\text { destination, } \\
\text { and final } \\
\text { disposal }\end{array}$ & $\begin{array}{l}\text { Medium and } \\
\text { low priority }\end{array}$ & $\begin{array}{l}\text { Environmental } \\
\text { and Economic }\end{array}$ \\
\hline $\begin{array}{l}\text { Destination } \\
\text { of house- } \\
\text { hold waste }\end{array}$ & $\begin{array}{l}\text { Nader, Salloum and } \\
\quad \text { Karam (2008) }\end{array}$ & Destination & $\begin{array}{l}\text { Medium and } \\
\text { low priority }\end{array}$ & Environmental \\
\hline $\begin{array}{l}\text { Existence } \\
\text { of recycling } \\
\text { companies }^{5}\end{array}$ & Martins and Cândido (2015) & Destination & $*$ & $*$ \\
\hline $\begin{array}{l}\text { Recycling } \\
\text { rate }^{6}\end{array}$ & $\begin{array}{l}\text { Mcalpine and Birnie (2005); } \\
\text { Fraser et al. (2006); Egil- } \\
\text { mez, Gumus, and Kucukvar } \\
\text { (2015); Lee and Huang } \\
\text { (2007); Tanguay et al. } \\
\text { (2010); Turcu (2012); } \\
\text { Turcu (2013); Cox et al. } \\
\text { (2010); Choi and Sir- } \\
\text { akaya (2006); Fehr et al. } \\
\text { (2014); Graymore (2014) }\end{array}$ & Destination & Medium priority & $\begin{array}{l}\text { Environmental, So- } \\
\text { cial and Economic }\end{array}$ \\
\hline $\begin{array}{c}\text { Recycled } \\
\text { waste volume }\end{array}$ & $\begin{array}{l}\text { Chávez-Cortés and } \\
\text { Maya (2010); Sui-Qui } \\
\text { and Leng (2015) }\end{array}$ & Destination & Medium priority & $\begin{array}{l}\text { Environmental, So- } \\
\text { cial and Economic }\end{array}$ \\
\hline $\begin{array}{l}\text { Number of } \\
\text { recycling } \\
\text { stations }\end{array}$ & $\begin{array}{l}\text { Robati, Monavari and } \\
\text { Majedi (2015) }\end{array}$ & Destination & Medium priority & Environmental \\
\hline $\begin{array}{c}\text { Ratio of } \\
\text { solid waste } \\
\text { composted to } \\
\text { total waste } \\
\text { production }\end{array}$ & Lee and Huang (2007) & Destination & Medium priority & $\begin{array}{l}\text { Environmental } \\
\text { and Economic }\end{array}$ \\
\hline
\end{tabular}




\begin{tabular}{|c|c|c|c|c|}
\hline $\begin{array}{l}\text { Recovery rate } \\
\text { of municipal } \\
\text { waste from } \\
\text { households }\end{array}$ & $\begin{array}{l}\text { Mickwitz and Mel- } \\
\text { anen (2009) }\end{array}$ & Destination & Medium priority & $\begin{array}{l}\text { Environmental, So- } \\
\text { cial, and Economic }\end{array}$ \\
\hline $\begin{array}{l}\text { Industrial } \\
\text { waste treat- } \\
\text { ment rate }\end{array}$ & Zhou et al. (2015) & Destination & Low priority & Environmental \\
\hline $\begin{array}{l}\text { Proportion of } \\
\text { construction } \\
\text { and demoli- } \\
\text { tion waste } \\
\text { treated }\end{array}$ & $\begin{array}{l}\text { Braulio-Gonzalo } \\
\text { et al. (2015) }\end{array}$ & Destination & Low priority & $\begin{array}{l}\text { Environmental } \\
\text { and Economic }\end{array}$ \\
\hline $\begin{array}{l}\text { Waste } \\
\text { treatment }\end{array}$ & Tourneau et al. (2013) & Destination & Low priority & $\begin{array}{l}\text { Environmental } \\
\text { and Economic }\end{array}$ \\
\hline $\begin{array}{l}\text { Waste } \\
\text { recovery and } \\
\text { disposal }\end{array}$ & $\begin{array}{l}\text { Mascarenhas, Nunes } \\
\text { and Ramos (2014) }\end{array}$ & $\begin{array}{l}\text { Destination } \\
\text { and final } \\
\text { disposal }\end{array}$ & $\begin{array}{l}\text { Medium and } \\
\text { low priority }\end{array}$ & $\begin{array}{l}\text { Environmental, So- } \\
\text { cial and Economic }\end{array}$ \\
\hline $\begin{array}{c}\text { Valorisation } \\
\text { and disposal } \\
\text { of waste }\end{array}$ & Mascarenhas et al. (2010) & $\begin{array}{l}\text { Destination } \\
\text { and final } \\
\text { disposal }\end{array}$ & $\begin{array}{l}\text { Medium and } \\
\text { low priority }\end{array}$ & $\begin{array}{l}\text { Environmental, So- } \\
\text { cial and Economic }\end{array}$ \\
\hline $\begin{array}{c}\text { Solid waste } \\
\text { disposal }\end{array}$ & Kelly and Moles (2002) & $\begin{array}{c}\text { Final } \\
\text { disposal }\end{array}$ & Low priority & Environmental \\
\hline $\begin{array}{c}\text { Per capita } \\
\text { discharge of } \\
\text { wastewater }\end{array}$ & Choi and Sirakaya (2006) & $\begin{array}{c}\text { Final } \\
\text { disposal }\end{array}$ & Low priority & Environmental \\
\hline $\begin{array}{l}\text { Problems in } \\
\text { disposing of } \\
\text { household } \\
\text { wastes }\end{array}$ & $\begin{array}{l}\text { Nader, Salloum and } \\
\quad \text { Karam (2008) }\end{array}$ & $\begin{array}{c}\text { Final } \\
\text { disposal }\end{array}$ & Low priority & $\begin{array}{l}\text { Environmental } \\
\text { and social }\end{array}$ \\
\hline $\begin{array}{c}\text { Elimination } \\
\text { of solid waste } \\
\text { in controlled } \\
\text { garbage } \\
\text { dumps }\end{array}$ & $\begin{array}{l}\text { Tolón-Becerra, Lastra-Bravo } \\
\text { and Galdeano-Gómez (2010) }\end{array}$ & $\begin{array}{c}\text { Final } \\
\text { disposal }\end{array}$ & Low priority & Environmental \\
\hline $\begin{array}{l}\text { Existence of } \\
\text { waste treat- } \\
\text { ment units }\end{array}$ & Martins and Cândido (2015) & $\begin{array}{c}\text { Final } \\
\text { disposal }\end{array}$ & Low priority & Environmental \\
\hline $\begin{array}{l}\text { Quantity of } \\
\text { household } \\
\text { waste sent } \\
\text { to landfills }{ }^{7}\end{array}$ & $\begin{array}{l}\text { Mickwitz and Melanen } \\
\text { (2009); Graymore (2014); } \\
\text { Tanguay et al. (2010) }\end{array}$ & $\begin{array}{c}\text { Final } \\
\text { disposal }\end{array}$ & Low priority & Environmental \\
\hline $\begin{array}{c}\text { Proportion of } \\
\text { solid waste } \\
\text { processed } \\
\text { by landfill }\end{array}$ & Manitiu and Pedrini (2016) & $\begin{array}{c}\text { Final } \\
\text { disposal }\end{array}$ & Low priority & Environmental \\
\hline
\end{tabular}




\begin{tabular}{|c|c|c|c|c|}
\hline $\begin{array}{c}\text { Prior- } \\
\text { ity of landfill } \\
\text { diversion }\end{array}$ & Fehr et al. (2014) & $\begin{array}{c}\text { Final } \\
\text { disposal }\end{array}$ & Low priority & Environmental \\
\hline $\begin{array}{c}\text { Landfill in } \\
\text { accordance } \\
\text { with standart }\end{array}$ & Sui-Qui and Leng (2015) & $\begin{array}{c}\text { Final } \\
\text { disposal }\end{array}$ & Low priority & Environmental \\
\hline $\begin{array}{l}\text { Landfill } \\
\text { quality }\end{array}$ & Fehr et al. (2014) & $\begin{array}{c}\text { Final } \\
\text { disposal }\end{array}$ & Low priority & Environmental \\
\hline $\begin{array}{l}\text { Solid waste } \\
\text { landfill area }\end{array}$ & Spilanis et al. (2009) & $\begin{array}{c}\text { Final } \\
\text { disposal }\end{array}$ & Low priority & Environmental \\
\hline $\begin{array}{l}\text { Period of in- } \\
\text { volvement in } \\
\text { recycling } \\
\text { programme }\end{array}$ & Choon et al. (2013) & Governance & * & Social \\
\hline $\begin{array}{c}\text { Existence } \\
\text { of commu- } \\
\text { nity recycling } \\
\text { projects }\end{array}$ & Martins and Cândido (2015) & Governance & * & Social \\
\hline $\begin{array}{c}\text { Type of } \\
\text { administration } \\
\text { (solid waste } \\
\text { management) }\end{array}$ & Fehr et al. (2014) & Governance & $*$ & $*$ \\
\hline $\begin{array}{l}\text { Submission } \\
\text { of recycling } \\
\text { programme } \\
\text { report }\end{array}$ & Choon et al. (2013) & Governance & $*$ & $*$ \\
\hline $\begin{array}{l}\text { Availabil- } \\
\text { ity of waste } \\
\text { management } \\
\text { and policy }\end{array}$ & Choi and Sirakaya (2006) & Governance & $*$ & $*$ \\
\hline $\begin{array}{c}\text { Forwards } \\
\text { waste to other } \\
\text { counties }\end{array}$ & Martins and Cândido (2015) & * & Low priority & $\begin{array}{l}\text { Environmental, So- } \\
\text { cial and Economic }\end{array}$ \\
\hline
\end{tabular}

Source: elaborated by the author

Notes:

1Including indicators "Access to regular solid waste collection (\%)" and "Percentage of area that receive garbage collection services".

Including the indicator "Population percentage assisted from two to three times per week by waste collection services".

3Including the indicator "Amount of hospital waste per capita (hab./day)".

4Including the indicators: "Existence of selective waste collection system" and "Municipal waste selectively collected".

5Including indicators "Existence of technology companies for material recycling" and "Existence of 
technologies patented for recycling”.

6Including indicators "Recycled municipal waste", "Proportion of recycled waste", "Amount of recycled waste (ton./hab./year)" and "Domestic waste recycling".

7Including indicator "Amount of domestic waste sent to landfills (ton./year)".

8Including indicators "Annual waste production", "Domestic waste production", "Waste generation and management", "Municipal solid waste generation", "Waste production per capita”, "Daily waste production", "Domestic waste", "Commercial waste”, "Amount of domestic solid waste per capita (hab./day)". 9Including indicator "Mean daily garbage collection per person".

*It was not possible classifying articles based on the available information.

The first observation lies on the fact that SW types (see Chart 1) were incorporated to IS. Although most indicators referred to domestic, commercial, and institutional waste, there are specific indicators for urban cleaning waste (for example, 'Construction and demolition waste per capita', industrial waste (for example, Industrial waste treatment rate) and health service waste. There were no specific indicators for agricultural or mining waste, which could be incorporated to locations, whose economic activities are based on the aforementioned activities. It brings back the discussion that this topic must be considered in a localized way, since there is great variability in waste generation, in quantitative and qualitative terms.

SWM is complex, it involves many stages (generation, collection, destination, final disposal, and governance) and how all these stages are interconnected to local sustainability. It is interesting that the set of LSA indicators aim at representing them (see Section 2). However, results have shown that only two systems (YUAN et al., 2003; MORENO PIRES; FIDÉLIS; RAMOS, 2014) count on indicators capable of capturing three stages in the SWM process. The set of indicators proposed by most systems $(94.3 \%)$ can represent only one or two of these stages. As it is described below, there is a trend of using solid waste destination, final disposal, and collection indicators; governance indicators were the least frequent ones.

Indicators of the SW "destination" stage were more frequent (30\%), with emphasis on indicator 'recycling rate', which is addressed in eleven sustainable assessment systems. It is important highlighting that Turcu $(2012 ; 2013)$ and Egilmez, Gumus and Kucukvar (2015) only used this indicator to represent the solid waste topic in the LSA system. It is understood that 'Recycling rate' approaches the environmental (in aspects regarding natural resources reduction and pollution prevention) and economic dimensions, which are mainly linked to the circular economy. Yet, the aforementioned indicator captures the social dimension related to the necessary move and commitment of population with the success of recycling programs. Although the three sustainability dimensions can be represented by this indicator, it can only depict intermediate hierarchy levels in solid waste management (recycling and waste acknowledgement), because it encompasses prior waste generation prevention and reduction matters.

Fourteen indicators (28\%) for stage 'final disposal' were identified and most of them 
only approach the environmental dimension, mainly pollution prevention aspects, such as 'Per capita discharge of solid waste' and 'Quantity of household waste sent to landfills'. There are three exceptions: indicator 'Problems in disposing of household wastes', which allows identifying the social dimension related to population well-being. Indicators 'Waste recovery and disposal' and 'Valorisation and disposal of waste', which show other environmental dimension aspects related to reducing the use of natural resources, besides pollution prevention. The same two indicators can be destined to represent the economic dimension referring to circular economy and social dimension, based on the population commitment aspect.

Ten indicators were also identified (20\%) and used to represent waste 'generation'. Authors Yigitcanlar and Dur (2010), King (2016) and Xu and Coors (2012) only used indicators of this nature to represent the waste issue. These authors understand that they are of paramount importance to represent the solid waste issue, because waste generation is intrinsically bond to consumption and, in the contemporary context, this aspect represents an important element for sustainability assessment. Actually, there is intense waste production, consumption and discharge flow (GONÇALVES-DIAS, 2015), which has negative impact on local sustainability. However, there are other sustainability aspects related to SW that can be captured through these indicators (for example, pollution prevention and democratic and participatory socio-environmental governance).

Indicators ability to represent SWM hierarchy importance levels was another assessed feature (high priority: prevention and non-generation; mean priority: reuse actions, recycling, and waste valorization; low priority: treatment actions or final disposal). Viable indicators that depict the low priory level were more frequent (40\%) and followed by the viable ones presenting high (18\%) and mean priority (14\%). Only five (10\%) ISs count on a set of indicators capable of depicting three SWM hierarchy levels, nineteen (38\%) of them can address two levels and eleven (24\%) only presented one level. Therefore, there was preference for using indicators and systems focused on waste post-generation. However, based on a sustainability perspective (since they are LSA studies), more indicators destined to measure society's efforts to prevent and minimize waste or reduce the demand for natural resources were expected (PELLETIER, 2010).

With respect to sustainability dimensions, most systems encompass indicators capable of representing aspects of three dimensions ( $72.22 \%$ of ISs). There are more indicators destined to capture and report environmental dimension aspects ( 40 indicators), namely: natural resources reduction and pollution prevention. As for the economic dimension, most indicators concern circular economy; however, two concepts were observed: 'Forwards waste to other counties' and 'Efficient collection system'. Such indicators regard a financial efficiency viewpoint. On the other hand, indicator 'Construction and demolition waste per capita' can indicate cleaner economic and industrial production processes. In total, 19 indicators can represent the aspects of the social dimension of sustainability: a) eleven indicators incorporate society mobilization to value, recover and recycle waste; b) four of them, 'Coverage of garbage collection', 'Frequency of collection', 'Problems in disposing of household wastes' and 'Number and \% of households experiencing problems 
in disposing of household wastes", concern society's well-being - besides public health issues caused by inappropriate waste disposal, such as discomfort caused by bad smell, visual contact and proliferation vectors (ZHANG; MATSUTO, 2013; SIMSEK et al., 2014) -; c) indicators 'Production of Municipal solid waste by type of waste', 'Collected solid waste per inhabitant and year', 'Composition of municipal waste' and 'Waste generation' draws light on the concept of social justice. The composition and amount of waste generated by society are intrinsically related to income, culture, and consumption pattern. Thus, it is possible carrying out an analysis about intra-generational (developed vs. developing countries, North vs. South, urban vs. rural population) and intergenerational equity.

The last analysis is related to the impact of the indicator-construction approach (participatory vs. non-participatory) that has the potential to represent SWM process stages, SWM hierarchy levels and sustainability dimensions. In total, $27.78 \%$ of the participatory LSAs presented a set of indicators capable of capturing at least three SWM elements, this ratio was lower in non-participatory LSA (12.5\%). Non-participatory system targets count on indicators to only represent one SWM stage; it happens, at smaller sphere, in participatory processes $(27.8 \%)$. By analyzing the representation of the SWM hierarchy order level, one can observe greater ratio of participatory IS captured by the other two lower levels (72.2\%) than the non-participatory IS (56.62\%). With respect to sustainability dimensions that must be the object of indicators, $77.78 \%$ of ISs based on participatory processes, have proposed indicator sets capable of representing the aspects of three sustainability dimensions; this ratio was lower in non-participatory IS (62.5\%).

\section{Final remarks}

Understanding how solid waste was inserted in local sustainability assessment systems is quite relevant to allow new systems to be built and to learn from existing limitations and potentials. The herein carried out review aimed at identifying and analyzing how indicators are used to represent the SW topic, which is of high complexity for private and public managers' decision-making.

The investigation showed that more than $80 \%$ of ISs and LSAs presented at most three indicators to represent all the complexity of SW. Accordingly, the set of indicators was not capable of capturing and representing all SWM stages. Results point out that most ISs $(80.5 \%)$ can represent, at most, these two stages. The SWM stages were organized in priory levels (SWM hierarchy); results have shown that the most used indicators aim at illustrating low priority levels (only five ISs count on a set of indicators capable of reporting the three levels). With respect to sustainability dimensions, $72.22 \%$ of ISs count on indicators capable of capturing aspects of the three dimensions (economic, environmental, and social). There was evidence that the participatory process has positive impact on the ability of the sets of indicators to represent SWM complexity and its connection to sustainability.

Some of the herein drawn general recommendations include more efficient SW indicators in indicator systems for local sustainability assessment: 
a) Based on sustainability principles, through its SW indicators, IS is recommend to capture aspects of natural resources used to reduce and prevent pollution (environmental dimension); adaptation to circular economy, financial efficiency and cleaner production (economic dimension); and well-being and social justice (social dimension);

b) It is recommended that ISs must approach all SWM hierarchy importance levels, from the use of technologies featured as 'end of pipe' to paradigmatic non-generation matters. Based on IS meta-analysis, there is maturation trend interacting with SWM; more and more lesser indicators of the SWM hierarchy order must be used;

c) It is recommended that all SWM process stages must be approached, because all of them are associated with sustainability aspects.

One of the lines for further studies may lie on a set of indicators common to SWM to integrate local IS. This common line will always be completed by the specificities of each location, mainly if waste is a critical factor about the SW indicators with indicators of other sustainability fields within IS, under integrated assessment approach (nonreductionist and fragmented into pillars subjected to the trade-offs effects).

\section{Acknowledgment}

This study was financed in part by the Coordenação de Aperfeiçoamento de Pessoal de Nível Superior - Brasil (CAPES) - Finance Code 001.

\section{References}

ADAMOVIĆ, V. M. et al. Prediction of municipal solid waste generation using artificial neural network approach enhanced by structural break analysis. Environ Sci Pollut Res, v. 24, p. 299-311, 2017.

BAGCH, A. Design of Landfills and Integrated Solid Waste Management. Wiley, 2004.

BARR, S.; GILG, A.; SHAW, G. 'Helping People Make Better Choices': Exploring the behaviour change agenda for environmental sustainability. Applied Geography, v.31, n.2, p. 712-720, 2011.

BJERKLI, C. L. Power in waste: Conflicting agendas in planning for integrated solid waste management in Addis Ababa, Ethiopia. Norwegian Journal of Geography, v. 69, n.1, p. 18-27, 2015

BELL, S.; MORSE, S. Breaking through the glass ceiling: who really cares about sustainability indicators? Local Environment, v.6, n.3, p. 291-309, 2001.

BOSSEL H. Indicators for Sustainable Development: Theory, Method, Applications. A Report to the Balaton Group. IISD, Canada. CES, 1999. Index of Sustainable and Economic Welfare. 
BRAULIO-GONZALO, M.; BOVEA, M. B.; RUÁ, M. J. 2015. Sustainability on the urban scale: Proposal of a structure of indicators for the Spanish context. Environmental Impact Assessment Review, v.53, p. 16-30, 2015.

BUENO, G.; LATASA, I.; LOZANO, P.J. Comparative LCA of two approaches with different emphasis on energy or material recovery for a municipal solid waste management system in Gipuzkoa. Renewable and Sustainable Energy Reviews, v.51, p. 449-459, 2015.

BULKELEY, H.; GREGSON, N. Crossing the Threshold: Municipal Waste Policy and Household Waste Generation. Environment and Planning A: Economy and Space, v. 41, p. 929-945, 2009.

BURNLEY, S.J. A review of municipal solid waste composition in the United Kingdom. Waste Management, v. 27, n. 10, p. 1274-1282, 2007.

CANAVESI, D.; ORTEGA, N. R. S.; QUEIRÓS, M. The assessment of local sustainability using fuzzy logic: An expert opinion system to evaluate environmental sanitation in the Algarve region, Portugal. Ecological Indicators, v.36, p. 711-718, 2014.

CASTELLANI, V.; SALA, S. 2010. Sustainable performance index for tourism policy development. Tourism Management, v.31, n.6, p. 871-880, 2010.

CASTELANI, V.; SALA, S. Sustainability Indicators Integrating Consumption Patterns in Strategic Environmental Assessment for Urban Planning. Sustainability, v. 5, p. 3426-3446, 2013.

CASTRO, M. A. O.; SILVA, N. M.; MARCHAND, G. A. E. L. Developing indicators for sustainable management of solid waste in Iranduba, Manacapuru and Novo Airão municipalities, Amazon, Brazil. Eng Sanit Ambient, v.20, n.3, p. 415-426, 2015.

CETRUlO, T. B. et al. Effectiveness of solid waste policies in developing countries: A case study in Brazil. Journal of Cleaner Production, v. 205, p.179-187, 2018.

CHAMIZO-GONZALEZ, J.; CANO-MONTERO, E. I.; MUÑOZ-COLOMINA, C.I. Municipal Solid Waste Management services and its funding in Spain. Resources, Conservation and Recycling, v. 107, p. 65-72, 2016.

CHÁVEZ-CORTÉS, M.; MAYA, J. A. A. 2010. Identifying and Structuring Values to Guide the Choice of Sustainability Indicators for Tourism Development. Sustainability, v. 2, p. 3074-3099, 2010.

CHOI, H. S. C.; SIRAKAYA, E. Sustainability indicators for managing community tourism. Tourism Management, v.27, n. 6, p. 1274-1289, 2006.

CHOON, S. W. et al. 2011. A sustainable city index for Malaysia. International Journal of Sustainable Development $\&$ World Ecology, v.18, p. 28-35, 2011.

CORVELLEC, H.; CAMPOS, M. J. Z.; ZAPATA, P. Infrastructures, Lock-in, and Sustainable Urban Development: The Case of Waste Incineration in the Göteborg Metropolitan Area. 
Journal of Cleaner Production, v. 50, p. 32-39, 2013.

COX, D. et al. Developing and using local community wellbeing indicators: Learning from the experience of Community Indicators Victoria. Australian Journal of Social Issues, v. 45, n.1, p. 71-88, 2010.

EGILMEZ, G.; GUMUS, S.; KUCUKVAR, M. 2015. Environmental sustainability benchmarking of the U.S. and Canada metropoles: An expert judgment-based multi-criteria decisionmaking approach. Cities, v. 42, p. 31-41, 2015.

ESMAEILIAN, B. et al. The future of waste management in smart and sustainable cities: A review and concept paper. Waste Management, v.81, p. 177-195, 2018.

EUROPEAN COMMISSION. (2008) Directiva 2008/98/CE, de 19 de noviembre de 2008 sobre los residuos y por la que se derogan determinadas Directivas. Diario Oficial de la Unión Europea.

FEHR, M. et al. Proposal of indicators to assess urban Sustainability in Brazil. Environment, Development and Sustainability, v. 6, p. 355-366, 2004.

FEREDAY, J.; MUIR-COCHRANE, E. Demonstrating Rigor Using Thematic Analysis: A Hybrid Approach of Inductive and Deductive Coding and Theme Development. International Journal of Qualitative Methods, v. 5, n. 1, 2006.

FRASER, E. D. G. et al. Bottom up and top down: Analysis of participatory processes for sustainability indicator identification as a pathway to community empowerment and sustainable environmental management. Journal of Environmental Management, v.78, n. 2, p. 114-127, 2006.

GIBSON, B.; HASSAN, S.; TANSEY, J. Sustainability Assessment: Criteria and Processes. London: Routledge, 2005.

GONÇALVES-DIAS, S. F. L. Consumo \& Resíduos: duas faces da mesma moeda. GV Executivo, v.14, n.1, 2015.

GRAYMORE, M. L. M. Sustainability Reporting: An Approach to Get the Right Mix of Theory and Practicality for Local Actors. Sustainability, v.6, n.6, p. 3145-3170, 2014.

GRUNERT, K. G.; THORGENSEN, J. Consumers, Policy and Environment: a tribute to Folke Ölander. New York: Springer, p.127-150, 2005.

GUERRERO, L. A.; MAAS, G.; HOGLAND, W. Solid waste management challenges for cities in developing countries. Waste Management, v. 33, n. 1, p. 220-232, 2013.

HENRIKSSON, G.; ÅKESSON, L.; EWERT, S. Uncertainty regarding waste handling in everyday life. Sustainability, n. 2, p. 2799-2813, 2010.

IACOVIDOU, E. et al. Metrics for optimising the multi-dimensional value of resources recovered from waste in a circular economy: A critical review. Journal of Cleaner Production, 
V. 166, p. 910-938, 2017.

ICLEI - Local Governments for Sustainability. About ICLEI. Disponível em: http://www.iclei. org/iclei-global/who-is-iclei. Acesso em: 04 dez 2018.

KAZA, S. et al. What a Waste 2.0: A Global Snapshot of Solid Waste Management to 2050 Urban Development Series. International Bank for Reconstruction and Development. The World Bank. 2018. Disponível em < https://openknowledge.worldbank.org/handle/10986/2174 $>$. Acesso em: 27 de Mar. de 2019.

KELLY, R.; MOLES, R. The Development of Local Agenda 21 in the Mid-west Region of Ireland: A Case Study in Interactive Research and Indicator Development. Journal of Environmental Planning and Management, v.45, n. 6, p. 889-912, 2002.

KING, L. K. Functional sustainability indicators. Ecological Indicators, v.66, p.121-131, 2016.

KRIPPENDORFF, K. Reliability in content analysis: some common misconceptions and recommendations. Human Communication Research, v. 30, n. 3, p. 411-433, 2004.

LEE, Y. J.; HUANG, C. M. Sustainability index for Taipei. Environmental Impact Assessment Review, v. 27, p. 505-521, 2007.

MANITIU, D. N.; PEDRINI, G. Urban smartness and sustainability in Europe. An ex ante assessment of environmental, social and cultural domains. European Planning Studies, 2016.

MAPAR, M. et al. Sustainability indicators for municipalities of megacities: Integrating health, safety and environmental performance. Ecological Indicators, n. 83, p. 271-291, 2017.

MARTINEZ-ALIER, J. Mining conflicts, environmental justice, and valuation. Journal of Hazardous Materials, v. 86, p.153-170, 2001.

MARTINS, M. F.; CÂNDIDO, G.A. Assessment model of urban sustainability level: proposal to Brazilian cities. Brazilian Journal of Urban Management, v.7, n.3, p. 397-410, 2015.

MASCARENHAS, A. et al. 2010. The role of common local indicators in regional sustainability assessment. Ecological Indicators, v.10, n.3, p. 646-656, 2010.

MASCARENHAS, A.; NUNES, L. M.; RAMOS, T. B. Selection of sustainability indicators for planning: combining stakeholders' participation and data reduction techniques. Journal of Cleaner Production, v. 92, p. 295-307, 2015.

MCALPINE, P.; BIRNIE, A. Is there a correct way of establishing sustainability indicators? The case of sustainability indicator development on the Island of Guernsey. Local Environment: The International Journal of Justice and Sustainability, v.10, n.3, p. 243-257, 2005.

MEADOWS, D. Indicators and informations systems for sustainable development. Hart Land Four Corners: The Sustainability Institute, 1998. 
MICKWITZ, P.; MELANEN, M. The role of co-operation between academia and policymakers for the development and use of sustainability indicators - a case from the Finnish Kymenlaakso Region. Journal of Cleaner Production, v. 17, n.12, p. 1086-1100, 2009.

MORENO PIRES, S. M. Sustainability Indicators and Local Governance in Portugal. 2011. Tese de Doutorado. Departamento de Ambiente e Ordenamento, Universidade de Aveiro, Aveiro, 2011.

MORENO-PIRES, S.; FIDÉLIS T, RAMOS TB. Measuring and comparing local sustainable development through common indicators: Constraints and achievements in practice. Cities, v. 39, p. 1-9, 2014.

NADER, M. R.; SALLOUM, B. A.; KARAM, N. Environment and sustainable development indicators in Lebanon: A practical municipal level approach. Ecological Indicators, v.8, n.5, p. $771-777,2008$.

NING, S. K.; CHANG, N. B.; HUNG, M. C. Comparative streamlined life cycle assessment for two types of municipal solid waste incinerator. Journal of Cleaner Production, v. 53, n. 15, p. 56-66, 2013.

PELLETIER, N. Of laws and limits: An ecological economic perspective on redressing the failure of contemporary global environmental governance. Global Environmental Change, 20, 220-228, 2010.

PELlETIER, N.; MAAS, R.; GORALCZYK, M.; WOLF, A. Conceptual basis for development of the European Sustainability Footprint. Environmental Development, 9, 12-23, 2014.

PEREIRA, F. S.; VIEIRA, I. C.G. Expansão urbana da Região Metropolitana de Belém sob a ótica de um sistema de índices de sustentabilidade. Ambiente \& Água - An Interdisciplinary Journal of Applied Science, v. 11, n.3, p. 731- 744, 2016.

PLATA-DÍAZ, A. M. et al. Alternative management structures for municipal waste collection services: The influence of economic and political factors. Waste Management, v.34, n.11, p. 1967-1976, 2014.

POLIDO, A.; JOÃO, E.; RAMOS, T.B. Sustainability approaches and strategic environmental assessment in small islands: An integrative review. Ocean $\&$ Coastal Management, v. 96, p. $138-148,2014$.

PRICE, J. L.; JOSEPH, J.B. Demand Management - A basis for Waste Policy: A critical review of the applicability of the Waste Hierarchy in terms of achieving sustainable waste management. Sustainable Development, v. 8, p. 96-105, 2000.

RAMOS, T. B.; CAEIRO, S. Meta-performance evaluation of sustainability indicators. Ecological Indicators, v. 10, n. 2, p. 157-166, 2010.

RAMOS, T. B.; CAEIRO, S.; MELO, J.J. Environmental indicator frameworks to design and assess environmental monitoring programs. Impact Assessment and Project Appraisal, v. 
22, n.1, p. 47-62, 2004.

REED, M. S.; FRASER, E. D. G.; DOUGILL, A. J. An adaptive learning process for developing and applying sustainability indicators with local communities. Ecological Economics, v.59, n.4, p. 406-418, 2006.

RHYNER, C.R. et al. Management and Resource Recovery. Lewis Publishers: New York (1995)

ROBATI, M.; MONAVARI, S. M.; MAJEDI, H. 2015.Urban Environment Quality Assessment by Using Composite Index Model. Environmental Progress \& Sustainable Energy, v. 34, n.5, p. 1473-1480, 2015.

RØPKE, I. Theories of practice - New inspiration for ecological economic studies on consumption. Ecological Economics, v.68, n.10, p. 2490-2497, 2009.

ROUSTA, K.; DAHLÉN, L. Source separation of household waste; technology and social aspects. In Taherzadeh, M.J., Richards, T., Eds. Resource Recovery to Approach Zero Municipal Waste. CRC Press: Boca Raton, FL, USA; p. 61-77, 2015.

RYDIN, Y.; HOLMAN, N.; WOLF, E. Local Sustainability Indicators. Local Environment: The International Journal of Justice and Sustainability, v.8, n.6, p. 581 589, 2003.

SAMSON, M. Not Just Recycling the Crisis: Producing Value at a Soweto Garbage Dump. Historical Materialism, v.25, n.1, p. 36-62, 2017.

SANTIAGO, L. S.; DIAS, S. M. F. Matrix of sustainability indicators for the urban solid waste management. Eng Sanit Ambient, v.17 n.2, p. 203-212, 2012.

SANTOS, V. M. N.; BACCI, D. L. C. Proposta para governança ambiental ante os dilemas socioambientais urbanos. Estud. av., São Paulo, v. 31, n. 89, p. 199-212, Apr. 2017.

SAVINI, F. The economy that runs on waste: accumulation in the circular city. Journal of Environmental Policy $\&$ Planning, Taylor \& Francis (on line) p.1-18, 2019.

SHEN, L. Y. et al. The application of urban sustainability indicators - A comparison between various practices. Habitat International, v.35, n.1, p. 17-29, 2011.

SIMSEK, C.; ELCI, A.; GUNDUZ, O.; TASKIN, N. An improved landfill site screening procedure under NIMBY syndrome constraints. Landscape and Urban Planning, v. 132, p.1-15, 2014.

SINGH, R. H. et al. An overview of sustainability assessment methodologies. Ecological Indicators, v. 15, n.1, p. 281-299, 2012.

SPILANIS, I. Monitoring sustainability in insular areas. Ecological Indicators, v.9, p.179-187, 2009.

SUI-QUI, T.; LENG, H. Review on the Development of a Sustainability Indicator System in 
Agenda 21 for Tourism in Mexico. International review for spatial planning and sustainable development, v.3, n.2, p. 4-21, 2015.

TANGUAY, G. A. et al. Measuring the sustainability of cities: An analysis of the use of local indicators. Ecological Indicators, v. 10, n. 2, p. 407-418, 2010.

TCHOBANOGLOUS, G.; KREITH, F.; WILLIAMS, M.E. Introduction. In: TCHOBANOGLOUS, G.; KREITH, F. Handbook of solid waste management. 2 ed. New York, McGrawHill, 2002.

TOLÓN-BECERRA, A.; LASTRA-BRAVO, X.; GALDEANO-GÓMEZ, E. Planning and neo-endogenous model for sustainable development in Spanish rural areas. Int. J. Sustainable Society, v.2, n.2, p. 156-176, 2010.

TOURNEAU, F. M.; et al. Assessing the impacts of sustainable development projects in the Amazon: the DURAMAZ experiment. Sustain Sci, v. 8, p. 199-212, 2013.

TURCU, C. Local experiences of urban sustainability: Researching Housing Market Renewal interventions in three English neighborhoods. Progress in Planning, v. 78, p. 101-150, 2012.

TURCU, C. 2013. Re-thinking sustainability indicators: local perspectives of urban sustainability. Journal of Environmental Planning and Management, v.56, n.5, p. 695-719, 2013.

UNITED NATIONS. Sustainable Development Goals - United Nations, 2015. Disponível em: http://www.un.org/sustainabledevelopment/sustainable-development-goals/. Acesso em: 04 dez 2018.

UNITED NATIONS ENVIRONMENTAL PROGRAMME. Waste and Climate Change: Global trends and strategy framework. Division of Technology, Industry and Economics, 2010.

UNITED NATIONS ENVIRONMENT PROGRAMME. Towards a Green Economy: Pathways to Sustainable Development and Poverty Eradication, 2011.

VALENTIN, A.; SPANGENBERG, J.H. A guide to community sustainability indicators. Environmental Impact Assessment Review, v.20, n.3, p. 381-392, 2000.

VALLIN, I. C.; GONÇALVES-DIAS, S. L. F. The Double Burden of Environmental Injustice in a Female Waste Pickers Cooperative in Brazil. Journal Für Entwicklungspolitik, v. XXXV, n. 2/3, p. 116-143, 2019.

VEIGA, T. B. et al. Building sustainability indicators in the health dimension for solid waste management. Rev. Latino-Am. Enfermagem, v. 24, 2016.

VERGARA, S. E.; TCHOBANOGLOUS, G. Municipal Solid Waste and the Environment: A Global Perspective. Annu. Rev. Environ. Resour. v. 37, p.277-309, 2012

XU, Z.; COORS, V. 2012. Combining system dynamics model, GIS and 3D visualization in sustainability assessment of urban residential development. Building and Environment, v. 
47, p. 272-287, 2012.

YIGITCANLAR, T;; DUR, F. Developing a Sustainability Assessment Model: The Sustainable Infrastructure, Land-Use, Environment and Transport Model. Sustainability, v.2, p. 321-340, 2010.

YUAN, W. et al. Development of sustainability indicators by communities in China: a case study of Chongming County, Shanghai. Journal of Environmental Management, v. 68, n. 3, p. 253-261, 2003.

WARLENIUS, R.; PIERCE, G.; RAMASAR, V. MART Global Environmental Change, v. 30, p. 21-30, 2015.

WILLIAMS, P.T. Waste Treatment and Disposal. 2 ed. John Wiley and Sons, 2005.

WILSON, D.C.; RODIC, L.; SCHEINBERG, A.; VELIS, C.A.; ALABASTER, G. Comparative analysis of solid waste management in 20 cities. Waste Management $\mathbb{\&}$ Research, v. 30, p. 237-254, 2012.

ZACCARIELLO, L.; CREMIATO, R.; MASTELLONE, M.L. Evaluation of municipal solid waste management performance by material flow analysis: Theoretical approach and case study. Waste Management $\mathbb{E}$ Research, v.33, n.10, p. 871-885, 2015.

ZHANG, X.; MATSUTO, T. Assessment of internal condition of waste in a roofed landfill. Waste Management, v. 33, p.102-108, 2013. 


\section{Natália Molina Cetrulo}

$\square$ molinacetrulo@gmail.com

ORCiD: https://orcid.org/0000-0003-1247-7079
Submitted on: 11/03/2019

Accepted on: 28/03/2020

2020;23:e00283

\section{Tiago Balieiro Cetrulo}

ఐ tiagocetrulo@gmail.com

ORCiD: https://orcid.org/0000-0002-2961-1839

\section{Sylmara Lopes Francelino Gonçalves Dias}

๑sgdias@usp.br

ORCiD: https://orcid.org/0000-0001-6326-2129

\section{Tomás Barros Ramos}

$\square$ tabr@fct.unl.pt

ORCiD: https://orcid.org/0000-0002-8270-4022

How to cite: CETRULO, N. M. et al. Solid waste indicators in local sustainability assessment: a literature review. Ambiente \& Sociedade. São Paulo, v. 23, p. 1-31, 2020. 


\title{
Indicadores de resíduos sólidos em sistemas de avaliação de sustentabilidade local: uma revisão da literatura
}

\author{
Natália Molina Cetrulo \\ Tiago Balieiro Cetrulo \\ Sylmara Lopes Francelino Gonçalves Dias \\ Tomás Barros Ramos
}

São Paulo. Vol. 23, 2020

Artigo Original

\begin{abstract}
Resumo: O tema resíduos sólidos (RS) no contexto de sistemas de avaliação de sustentabilidade local (ASL) ainda foi pouco explorado. Este artigo faz uma revisão da literatura sobre essa temática, com foco na análise dos indicadores de RS e dos sistemas em que estão inseridos. É examinado se os indicadores conseguem representar a complexidade dos RS e aspectos da sustentabilidade. Os resultados desta revisão evidenciaram que $77,8 \%$ dos sistemas de indicadores utilizam no máximo dois indicadores para os RS. Evidenciaram também que a maioria deles é dedicada a representar as etapas da destinação e disposição final da gestão dos RS, os aspectos ambientais da sustentabilidade e os níveis de ordem intermediária na hierarquia de gestão de RS. Foram encontradas evidências sobre o impacto positivo da Participação pública nas questões analisadas. As considerações finais apresentam recomendações sobre a inclusão de indicadores de RS em sistemas de ASL.
\end{abstract}

Palavras-chave: Indicadores de sustentabilidade; Avaliação de sustentabilidade local; Participação pública; Hierarquia da gestão de resíduos.

Como citar: CETRULO, N. M. et al. Indicadores de resíduos sólidos em sistemas de avaliação de sustentabilidade local: uma revisão da literatura. Ambiente \& Sociedade. São Paulo, v. 23, p. 1-33, 2020.

DOI: http://dx.doi.org/10.1590/1809-4422asoc20190028r3vu2020L5AO 


\title{
Indicadores de residuos sólidos en sistemas locales de evaluación de sostenibilidad: una revisión de la literatura.
}

\author{
Natália Molina Cetrulo \\ Tiago Balieiro Cetrulo \\ Sylmara L. F. Gonçalves Dias \\ Tomás Barros Ramos
}

São Paulo. Vol. 23, 2020

Artículo original
Resumen: El tema resíduos sólidos (RS) dentro del contexto de evaluación de sostenibilidad local (ESL) aún fue poco explorado. Este artículo trae un revisión de literatura sobre esa temática, centrándose en el análisis de los indicadores de RS y los sistemas en los que se insertan. Es examinado si los indicadores pueden representar la complejidad de la RS y los aspectos de sostenibilidad. Los resultados de esta revisión evidencian que $77.8 \%$ de los SI utilizan como máximo 2 indicadores para los RS. Evidencian también que la mayoría de ellos es dedicado a representar los elementos de la destinación y disposición final de la gestión de los RS, los aspectos ambientales de sostenibilidad y los niveles de orden intermedio en la jerarquía de gestión de RS. Se encontró evidencia sobre el impacto positivo de la participación social en los temas analizados. Las consideraciones finales presentan recomendaciones sobre la inclusión de indicadores de RS en sistemas de ASL.

Palabras-clave: Indicadores de sostenibilidad, Evaluación de la sostenibilidad local ; Participación social; Jerarquía de gestión de resíduos.

Como citar: CETRULO, N. M. et al. Indicadores de residuos sólidos en sistemas locales de evaluación de sostenibilidad: una revisión de la literaturaa. Ambiente $\&$ Sociedade. São Paulo, v. 23, p. 1-33, 2020.

DOI: http://dx.doi.org/10.1590/1809-4422asoc20190028r3vu2020L5AO 